\title{
Insulin Injection A Non-Trivial Act with Sometimes Serious Consequences: Major Role of Therapeutic Education
}

\author{
R. Louzolo-Kimbembe , FZ. EL Jaafari, S. Rafi, G. El Mghari, N. El Ansari
}

Department of Endocrinology, Diabetology, Metabolic Diseases and Nutrition, Mohammed VI University Hospital of Marrakesh, Morocco

DOI: $10.36347 /$ sasjm.2021.v07i02.005

| Received: 08.02.2021 | Accepted: 19.02.2021 | Published: 21.02 .2021

*Corresponding author: Rolly Junior Louzolo-Kimbembe

Abstract

Case Report

Insulin, the more powerful antihyperglycemic agent, is the most widely used treatment in the management of diabetes mellitus. This product has undergone several developments since its invention, basically with the modification of its structure to allow better efficiency and form with the appearance of insulin pens, more convenient. Despite this, many people with diabetes fail to reach glycemic targets. The problem stems among other things from errors due to the technique of administration of insulin. These errors are numerous but underestimated because less elucidated but fortunately easily correctable once detected. This relies on good patient education on the injection technique, which may already partially resolve the diabetes imbalance.

Keywords: Insulin pens, antihyperglycemic agent, diabetes imbalance, injection technique.

Copyright $(\mathcal{C} 2021$ The Author(s): This is an open-access article distributed under the terms of the Creative Commons Attribution 4.0 International License (CC BY-NC 4.0) which permits unrestricted use, distribution, and reproduction in any medium for non-commercial use provided the original author and source are credited.

\section{INTRODUCTION}

In diabetic patients, insulin therapy is essential in the management of their diabetes, which can be permanent or temporary depending on whether it is type 1 (T1DM) or type 2 (T2DM) diabetes. However, rigorous asepsis is necessary to avoid any infection at the site of the insulin injection, which would constitute a gateway. Insulin therapy poorly conducted without following certain rules of injection and asepsis can have infectious consequences on the skin in the presence of contributing factors such as taking non-steroidal antiinflammatory drugs (NSAIDs), unbalanced diabetes, or other immunosuppression. This can lead to complications such as cellulitis which is an acute and extensive infection of the dermis and subcutaneous tissue, complicating a wound, ulcer, skin disease, or infection at the site of insulin injection, or an exceptional complication such as necrotizing fasciitis, which is a rare and serious infection of the skin and deep subcutaneous tissues with a tendency to rapid spread of infectious necrosis in the absence of urgent medical and surgical treatment. We report a case of infectious cellulitis of the abdominal wall and necrotizing fasciitis of the thigh following an injection of insulin.

\section{Case Report 1}

50-year-old patient, T2DM, on oral antidiabetic drugs, with recent addition of a semi-slow insulin at bedtime (bed time profile) without any other particular pathological history, presented an infectious cellulitis of the anterior abdominal wall following an insulin injection at this site. The anamnesis and the examination found, the notion of initial taking of NSAIDs, a red, hot, painful inflammatory patch on the level of the anterior face of the abdominal wall of $10 \mathrm{~cm}$ * $13 \mathrm{~cm}$, periumbilical, with a central ulceration and a yellowish deposit, painful and hard on palpation. 


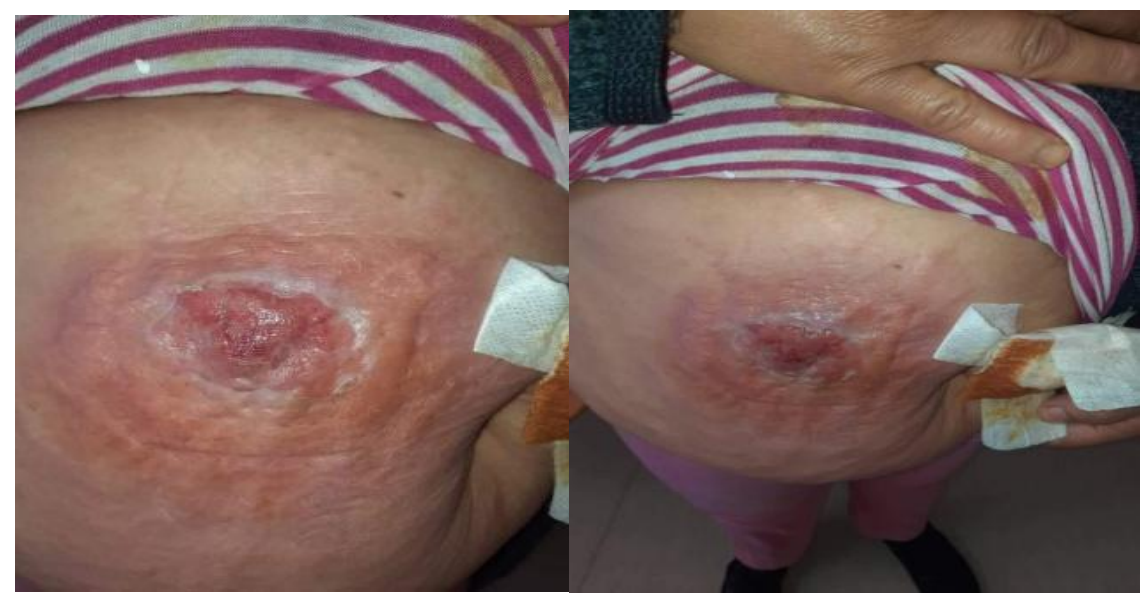

Fig-1: Cellulitis of the abdominal wall

\begin{abstract}
The clinical picture was associated with a significant infectious assessment, with hyperleukocytosis at $19.800 / \mathrm{mm} 3$ and CRP at $187 \mathrm{mg} /$ 1 with soft tissue ultrasound: an edematous infiltration of the soft parts of the abdominal wall. The patient was treated with antibiotic therapy and local care, with discontinuation of oral anti-diabetic drugs and put on intensified insulin therapy.
\end{abstract}

\section{Case Report 2}

A 19-year-old patient, T1DM for 2 years, under basal bolus, presented with swelling of the anterior aspect of the left thigh following an injection of insulin at this site. Initially put on probabilistic antibiotic therapy (protected amoxicillin) with the notion of taking NSAIDs. This resulted in a worsening of the picture towards necrotizing fasciitis which required emergency hospitalization. The examination found a swollen, painful left thigh with areas of necrosis as well as a significant infectious assessment with hyperleukocytosis at $21.000 / \mathrm{mm} 3$ and CRP at $194 \mathrm{mg}$ $/ 1$.

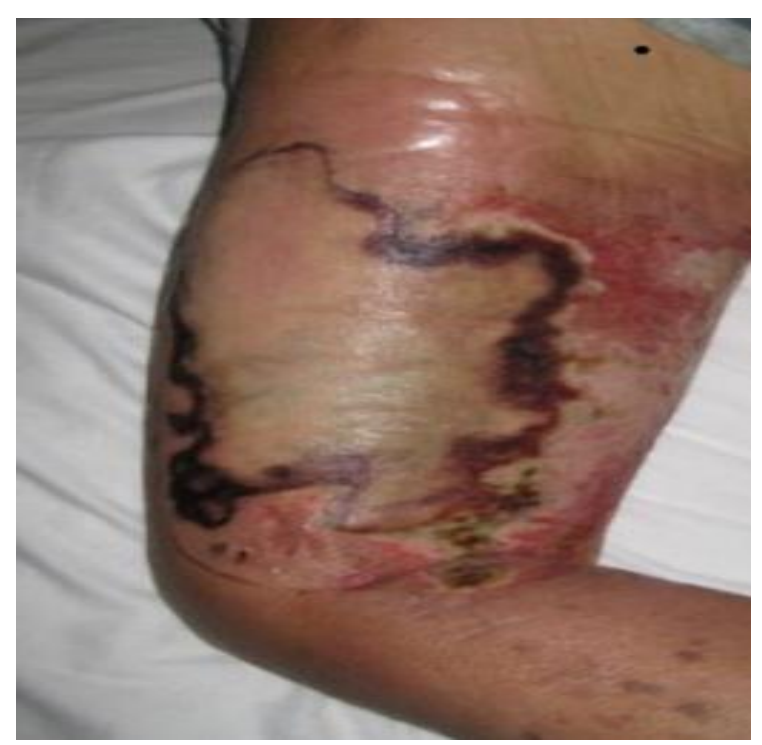

Fig-2: Progressive necrosis

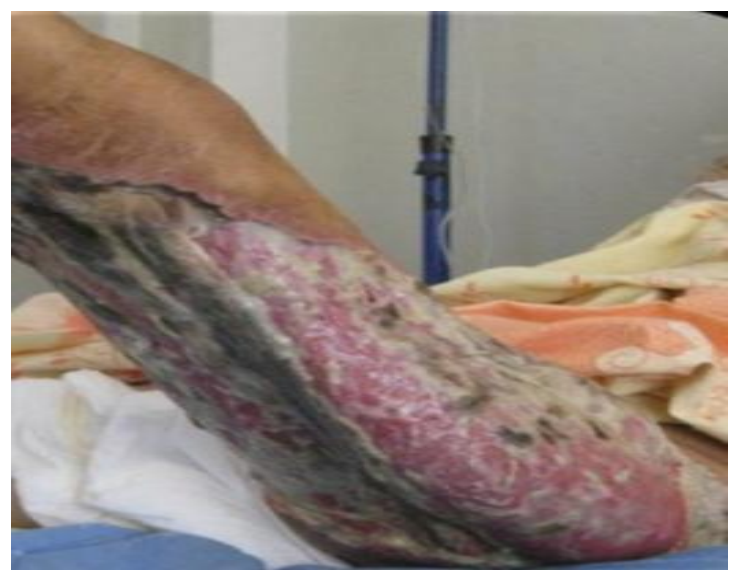

Fig-3: After necrosectomy

Emergency management consisted of necrosectomy with daily local care, antibiotic therapy adapted to the germ (streptococcus) and intensification of insulin therapy.

\section{DISCUSSION}

These two cases illustrate that negligent administration of insulin is not without consequences. In the first case, it was her first insulin injection for the DM2 patient, so she was insulin naïve and ignorant of any proper insulin injection technique. In the second case, the young DM1, long accustomed to insulin therapy, would have used the same syringe and therefore the same needle during several insulin injections. These two patients were not using an insulin analog pen but vials of human insulin drawn up with an insulin syringe. Although the administration of insulin by syringe is associated with a high risk of errors, in particular dosing errors, and despite the various advantages offered by pens, particularly in terms of user-friendliness and comfort of the injection, which facilitate the Insulin initiation errors in the technique of insulin delivery remain a problem with both the conventional and the modern method [2].

Those errors on insulin administration were revealed, in the first case by asking the patient to demonstrate how she performed her insulin injection 
and in the second by observing the deformation of the needle of the syringe used by the patient. Skin complications at the injection site are quite common following non-compliance with injection techniques and asepsis rules and insulin therapy can lead to skin complications such as in our two cases, an infectious subcutaneous abscess or necrotizing fasciitis which is rare.

But these complications can be avoided by therapeutic education aimed at acquiring skills on the theoretical aspects and technical skills of the gesture. In this sense, the use of insulin has several elements that must be taught and learned, including the storage of insulin, care at the injection site, the injection process and technique, skin folds, injection site rotation, needle use and length [1]. Other considerations that are taught when using insulin include injection complications, proper disposal of injection equipment, hypoglycemia, and monitoring. All information given to a patient during insulin initiation likely results in an inability to retain all of the information taught. It is therefore necessary to ensure the transfer of skills from the educator (health personnel) to the learner (patient) through appropriate educational tools during workshops on the rules and techniques of insulin injections and always make an objective evaluation over time of the acquisition of new self-care skills by the patient because a skill not assessed is a skill not learned.

This involves the need for active listening in order to identify gray areas and remedy them, patient support and permanent assessment of acquired knowledge, the need for total patient support in the management of their disease. Because not knowing or respecting the rules and techniques of injections result in infectious complications that can have a bad prognosis. We must therefore avoid the trivialization of the technical gesture, support the patient in his learning and correct the error. Thus the capacities to be acquired by the patient must be to respect the principles of hygiene and asepsis, to change the injection site, to always clean the injection site, not to carry out an injection through clothing., to change the site in the event of an infection nearby, to know the risks of reusing the same needle: the needle is no longer sterile and its bevel may become blunt, increasing the risk of microtrauma or even pain during injection, appearance of lipodystrophies with inadequate doses [4], with also the risk that the needle breaks and that a piece of metal remains under the skin.

Several studies have shown that errors in the rules and techniques for self-injection of insulin are due to patients' lack of knowledge of the different stages of administration of insulin [1]. The various errors reported in the literature relate to injecting at the same site, injecting into areas of lipodystrophies, reusing needles, injecting through clothing and not mixing cloudy insulins before use. Most patients report that they have not been educated or initiated in the techniques of insulin injections. However, it is difficult to know if patients have not learned these skills or if they have simply forgotten them due to all information given to them. Nevertheless, the educator or health worker is required to constantly assess and reassess skill acquisition, not hesitate to repeat educational messages until patients understand the technique and why it is important.

An effective approach used by health or educational personnel is the method of confirmation of communication. It confirms whether a patient understands what is explained to him by asking him to re-explain in his own words what he has learned after being introduced to the concept for the first time (teachback method). The feedback method also makes it possible to assess the effectiveness of the educator's capacity (health personnel) to transmit concepts to the learner (patient). It allows for immediate correction and clarification of skills and concepts if they are not well understood [1].

\section{CONCLUSION}

Although a priori simple, an insulin injection can, if it is not carried out under the strict rules of asepsis and with good technique, lead to more or less serious complications. Total patient support from the outset in the management of their disease is therefore essential. It involves a motivational counseling with support including a therapeutic education touching the dimensions of the person that improves their knowledge and their motivations to learn to take better care of themselves. This article reinforces the need for repeated patient education with assessment and reassessment of prior learning. The feedback method is a fundamental approach in which assessment and rehabilitation are carried out at every available opportunity to reduce complications and rate of hospitalization due to poor injection technique.

\section{REFERENCES}

1. Truong TH, Nguyen TT, Armor BL, Farley JR. Errors in the administration technique of insulin pen devices: a result of insufficient education. Diabetes Therapy. 2017 Apr;8(2):221-6.

2. Baruah MP. Insulin pens: the modern delivery devices. J Assoc Physicians India. 2011 Apr 1;59(Suppl):38-40.

3. Chowdhury S, Chakraborty PP. Errors of insulin therapy: Real-life experiences from developing world. Journal of family medicine and primary care. 2017 Oct;6(4):724-729.

4. Frid AH, Kreugel G, Grassi G, Halimi S, Hicks D, Hirsch LJ, Smith MJ, Wellhoener R, Bode BW, Hirsch IB, Kalra S. New insulin delivery recommendations. InMayo Clinic Proceedings 2016 Sep 1 (Vol. 91, No. 9, pp. 1231-1255). Elsevier. 